\title{
Reproducibility of the Adapted Leger and Boucher Test for wheelchair-dependent athletes
}

\author{
M Poulain*,1, A Vinet ${ }^{2}$, PL Bernard ${ }^{3}$ and A Varray ${ }^{1}$ \\ ${ }^{1}$ Laboratory 'Sports, Intervention, Optimization', School of Sports Sciences and Physical Education, 700 avenue du \\ Pic St Loup, 34090 Montpellier, France; ${ }^{2}$ Laboratory 'Sports, Health, Development', School of Sports Sciences and \\ Physical Education, 700 avenue du Pic St Loup, 34090 Montpellier, France; ${ }^{3}$ Laboratory of Biomechanics and \\ Exercise Biology, UFR STAPS, 261 route de Grenoble, F-06200, Nice, France
}

Study design: This study analyzed the reproducibility of a field test. In a previous study, we showed that this test, the Adapted Leger and Boucher Test (ALBT), was progressive and maximal. $^{1}$ The Leger and Boucher predictive equation for able-bodied subjects ${ }^{2}$ was not accurate for WD athletes, however, and a new predictive equation is needed.

Objectives: To determine the reproducibility of an adapted incremental field test for wheelchair-dependent (WD) athletes.

Setting: France at Montpellier.

Methods: The proposed protocol was conducted on a $400 \mathrm{~m}$ track. Eight male paraplegics (mean age: $30.8 \pm 5.1$ years) performed the test three times in the same conditions, ie same time of day, same wheelchair, same material. Maximal heart rate (HRmax) and maximal speed (Smax) were measured.

Results: We found no significant differences $(P>0.05)$ between tests for either variable. The Bland and Altman graphic analyses showed a good reproducibility for both variables. Lastly, the reproducibility coefficients of HRmax and Smax were very low ( $2 \%$ and $1 \%$, respectively). Conclusion: The ALBT is reproducible concerning measurements of HRmax and Smax. A valid predictive equation of maximal oxygen uptake from the Smax is now needed for WD athletes during this field test.

Keywords: paraplegia; incremental field test; reproducibility; maximal speed; Leger and Boucher Test

\section{Introduction}

Research in athletics is focused on the assessment of the factors that constitute performance. Based on the results of the Paralympic Games, one can conclude that wheelchair athletics is expanding and that these athletes would benefit from data geared toward optimizing their performance. For example, today's competitive wheelchair athletes need training programs that are based on regular evaluation of their aerobic metabolism in the wheelchair. Laboratory procedures for cardiorespiratory fitness assessment are now wellestablished, with different types of ergometer adapted for wheelchair propulsion. ${ }^{3-6}$ Wheelchair ergometers, however, do not accurately reproduce wheelchair propulsion conditions such as rolling resistance. Field testing has been shown to provide an accurate estimation of aerobic metabolism in practice conditions and is easy to conduct in able-bodied (AB) subjects. The different field tests are used regularly to evaluate maximal oxygen uptake $\left(\mathrm{VO}_{2} \mathrm{max}\right)$ in order to adapt training programs. The 12-min test of Cooper

*Correspondence: M Poulain, Cinique du Souffle 'la Solane', 19 rue des Casteillets, 66340 Osséja, France was validated ${ }^{7,8}$ in paraplegic subjects, but its nonprogressive character requires learning and the ability to establish a steady pace. Moreover, the subjects were evaluated with the same standardized wheelchair. Recently, we validated the Adapted Leger and Boucher Test (ALBT) in wheelchair-dependent (WD) athletes ${ }^{1}$ as a progressive and maximal test. However, the predictive equation, based only on maximal speed (Smax) for AB subjects, was not valid. The assumption was therefore made that other parameters, in addition to Smax, would be needed for a valid equation for WD athletes. The next step was thus to assess the reproducibility of this test in terms of: (1) maximal heart rate (HRmax), to verify the reproducibility of the maximal character of the test, and (2) maximal speed (Smax), to predict maximal oxygen uptake $\left(\mathrm{VO}_{2} \mathrm{max}\right)$.

\section{Methods}

\section{Subjects}

Eight male WD athletes, aged $30.8 \pm 5.1$ years (range 24 to 39 years) participated in this study after giving 
Table 1 General characteristics of WD athletes

\begin{tabular}{|c|c|c|c|c|c|c|c|c|}
\hline $\begin{array}{l}\text { Subjects } \\
(\mathrm{n}=8)\end{array}$ & $\begin{array}{l}\text { Age } \\
\text { (years }\end{array}$ & $\begin{array}{l}\text { Height } \\
(\mathrm{cm})\end{array}$ & $\begin{array}{c}\text { Date of } \\
\text { injury }\end{array}$ & $\begin{array}{l}\text { Lesion } \\
\text { level } \\
(T-L)^{*}\end{array}$ & $\begin{array}{c}\text { Lesion } \\
\text { type } \\
(I-C-S-F) * *\end{array}$ & $\begin{array}{c}\text { Sport } \\
(T F-T-T T) \dagger\end{array}$ & $\begin{array}{c}\text { Training } \\
\text { volume } \\
\left(h \text { week }^{-1}\right)\end{array}$ & Level: \\
\hline 1 & 31 & 171 & 1983 & $\mathrm{~T} 4$ & $\mathrm{C} / \mathrm{S}$ & $\mathrm{TF}$ & 3 & Marathon $\mathrm{R}$ \\
\hline 2 & 35 & 176 & 1980 & $\mathrm{~T} 8$ & $\mathrm{C} / \mathrm{S}$ & $\mathrm{T}$ & 5 & $\mathrm{~N}$ \\
\hline 3 & 28 & 180 & 1984 & L3 & $\mathrm{I} / \mathrm{F}$ & $\mathrm{T}$ & 6 & I \\
\hline 4 & 25 & 170 & 1988 & $\mathrm{~T} 12$ & $\mathrm{I}$ & $\mathrm{T}$ & 4 & I \\
\hline 5 & 39 & 170 & 1988 & T9 & $\mathrm{C} / \mathrm{F}$ & TT & 12 & I \\
\hline 6 & 24 & 175 & 1984 & $\mathrm{~T} 8$ & $\mathrm{C} / \mathrm{S}$ & $\mathrm{T}$ & 4 & $\mathrm{R}$ \\
\hline 7 & 34 & 175 & 1984 & $\mathrm{~T} 10$ & $\mathrm{C} / \mathrm{S}$ & $\mathrm{T}$ & 2 & $\mathrm{R}$ \\
\hline 8 & 30 & 170 & 1983 & $\mathrm{~T} 4$ & $\mathrm{I} / \mathrm{S}$ & $\mathrm{T}$ & 3 & $\mathrm{R}$ \\
\hline Mean & 30.8 & 173.37 & & - & - & - & $5 \mathrm{~h} 27 \mathrm{~min}$ & \\
\hline SD & 5.1 & 3.70 & & - & - & - & $3 \mathrm{~h} 13 \mathrm{~min}$ & \\
\hline
\end{tabular}

$* \mathrm{~T}=$ thoracic, $\mathrm{L}=$ lumbar; $* * \mathrm{I}=$ incomplete, $\mathrm{C}=$ complete, $\mathrm{S}=$ spastic, $\mathrm{F}=$ flaccid; $\dagger \mathrm{TF}=$ track and field, $\mathrm{T}=$ tennis, $\mathrm{TT}=$ table tennis; International classification, $\mathrm{R}=$ regional, $\mathrm{N}=$ national, $\mathrm{I}=$ international

informed written consent. All were paraplegics with lesion levels of T4-L3, stabilized from their injuries, and were classed according to the ISMG classification. ${ }^{9}$ Furthermore, all had spent a minimum of 2 years in a wheelchair. The athletes practiced track and field (marathon), tennis (regional, national and international competitors) or table tennis (national competitor). They had acquired good control of their wheelchairs, which was essential for the test, and they were used to all-out effort. The general characteristics of the subjects are shown in Table 1.

\section{Protocol}

Each subject performed the ALBT on three separate occasions. The tests were scheduled for the same time of day, with the same material, and in verified comparable weather conditions, especially concerning wind speed and temperature. For the analysis of short-term reproducibility (STR), we established a minimal interval of 3 days and a maximal interval of 10 days between the first and second tests. For the analysis of long-term reproducibility, the intervals ranged from a minimal period of 8 days to a maximal of 1 month between the first and third tests (LTR). This maximal period was chosen to avoid the risk of any significant modification in the subject's physical fitness.

The ALBT was conducted on a $400 \mathrm{~m}$ tartan field marked-off every $50 \mathrm{~m}$ with pylons. The wind speed was measured with an anemometer prior to testing and had to be less than $2 \mathrm{~m} \mathrm{~s}^{-1}$. The test protocol required each subject to propel his wheelchair as far as possible following the rhythm imposed by means of an audiotape that provided feedback to the subjects. The turning speed of the tape recorder was checked prior to the start of each test to ensure that any deviation was less than $1 \mathrm{~s} \mathrm{~min}^{-1} .{ }^{10}$ The athletes adjusted their speed as necessary when crossing a pylon at the sound signal. There was only a general warm-up, which was included in the test. The initial speed was $4 \mathrm{~km} \mathrm{~h}^{-1}$
Table 2 Individual HRmax and variability coefficients for the three tests

\begin{tabular}{lcccc}
\hline & \multicolumn{3}{c}{ HRmax (beats.min ${ }^{-1}$ ) } & Var coef \\
Subjects & First test & Second test & Third test & HRmax \\
\hline 1 & 173 & 170 & 175 & 0 \\
2 & 195 & 195 & 195 & 0 \\
3 & 195 & 187 & 185 & 0.03 \\
4 & 190 & 168 & 184 & 0.03 \\
5 & 171 & 175 & 174 & 0.04 \\
6 & 171 & 174 & 182 & 0.03 \\
7 & 159 & 177 & 169 & 0.03 \\
8 & 195 & 193 & 188 & 0 \\
Mean & 181.1 & 179.8 & 181.5 & 0.02 \\
SD & 14.22 & 10.39 & 8.43 & 0.01 \\
\hline
\end{tabular}

Var $\operatorname{coef}=$ variability coefficient

for $1 \mathrm{~min}$; thereafter it was increased by $1 \mathrm{~km} \mathrm{~h}^{-1}$ every minute until exhaustion, which was defined as the inability to maintain the required speed with a $3-\mathrm{m}$ distance behind the appropriate pylon at the sound signal and the inability to catch up at the next pylon. The number of executed laps, the number of crossed pylons and the duration of the test were noted. The last speed to be reached and maintained $1 \mathrm{~min}$ corresponded to the Smax defined as the speed necessary to attain $\mathrm{VO}_{2}$ max. ${ }^{2,11}$ Throughout the test, HR was measured every $15 \mathrm{~s}$ with a cardiofrequency meter (Sport Tester PE3000, Polar, Finland). The theoretical maximal HR $[210-(0.65 \times \text { age })]^{12}$ was calculated to determine whether subjects reached their maximal values.

\section{Statistical analysis}

Specific contrasts were calculated to determine whether differences existed between the theoretical HRmax ${ }^{12}$ and the HRmax obtained at the three tests. Results were considered to be significant when $\alpha$ risk was less than 0.05. A one-way repeated-measures analysis of 
variance (MANOVA), realized with SYSTAT 5.02, was used to examine the differences between means (SD) of both Smax and HRmax and temperature. The Bland and Altman procedure ${ }^{13}$ and the calculation of reproducibility coefficients (difference/mean) were used to test the reproducibility of the ALBT for HRmax
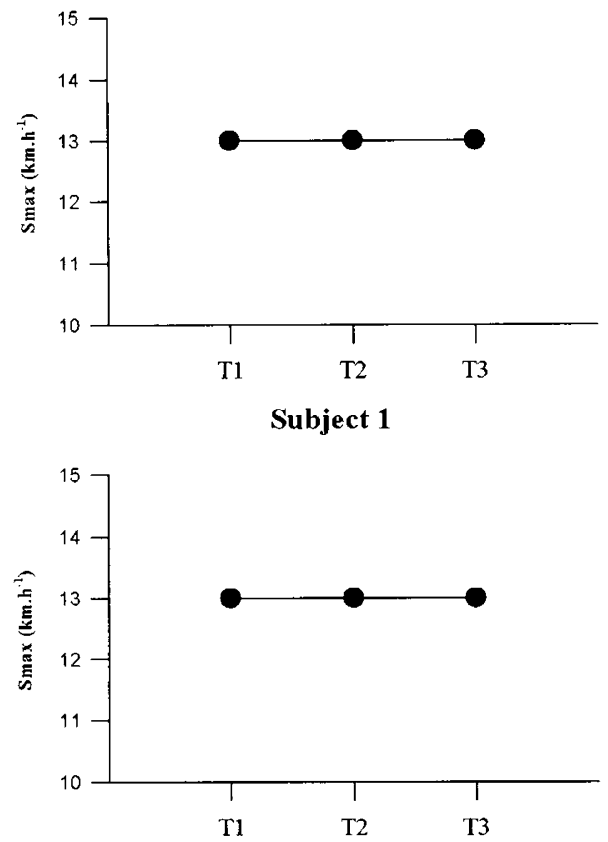

Subject 2
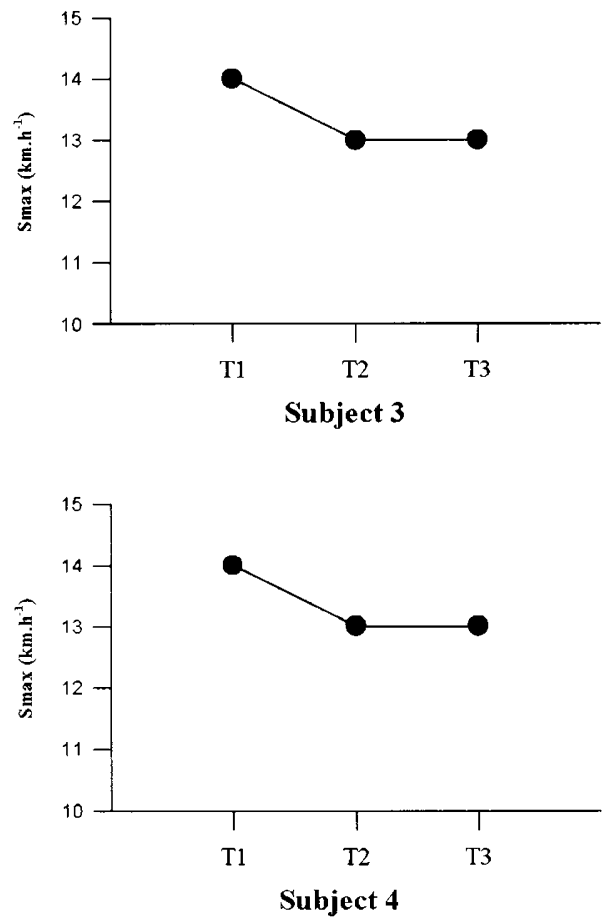

and Smax. For the Bland and Altman procedure, we plotted the difference of two measurements, ie between the first and the second test (STR) and the first and the third test (LTR), for each subject against his mean value. Results are reported as mean \pm standard deviation.
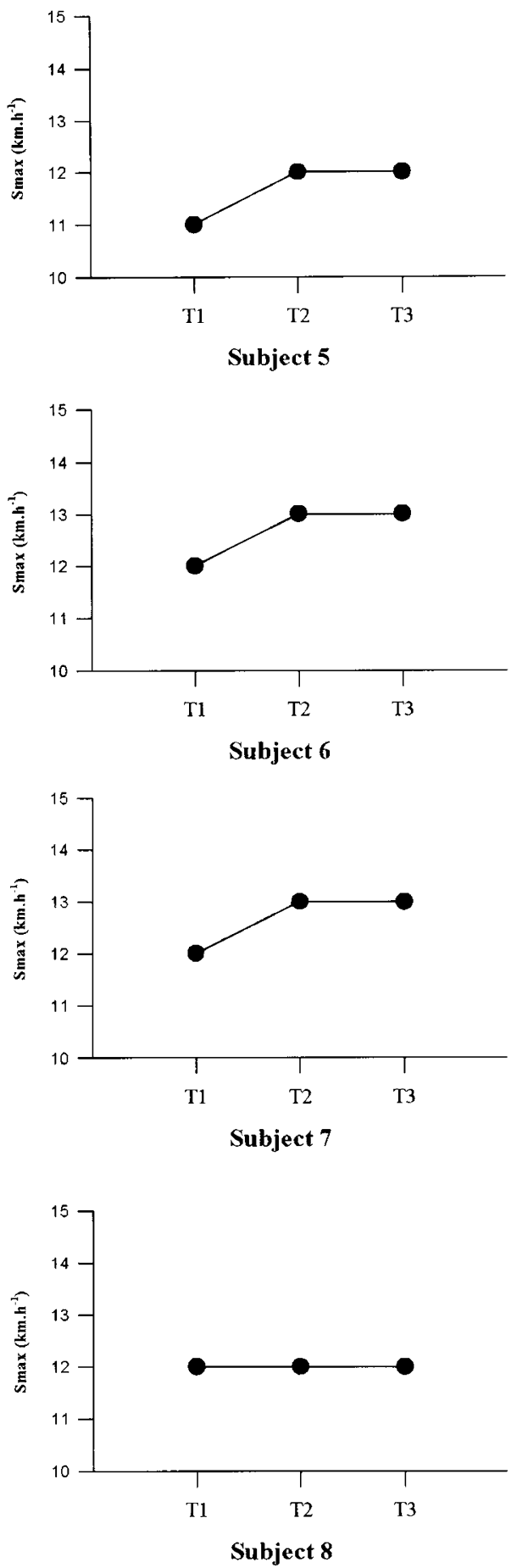

Figure 1 Maximal speed (Smax in $\mathrm{km} \mathrm{h}^{-1}$ ) obtained during the three tests in each subject 


\section{Results}

The values of theoretical HRmax ranged from 184.5 to 194.4 beats $\min ^{-1}$. The values of HRmax (Table 2) of the ALBT ranged from 168 to 195 beats $\min ^{-1}$. The mean value was $180.83 \pm 9.98$, which represents $95.2 \% \pm 5.4$ of theoretical HRmax. The theoretical
HRmax compared with the HRmax obtained during the tests presented no significant difference $(F=3.59$, $P=0.10)$.

The mean temperature in degrees Celsius was $22.1^{\circ} \pm 2.9$ and there was no significant difference between the three tests $(F=0.04, \quad P=0.95)$. No significant differences were observed between the
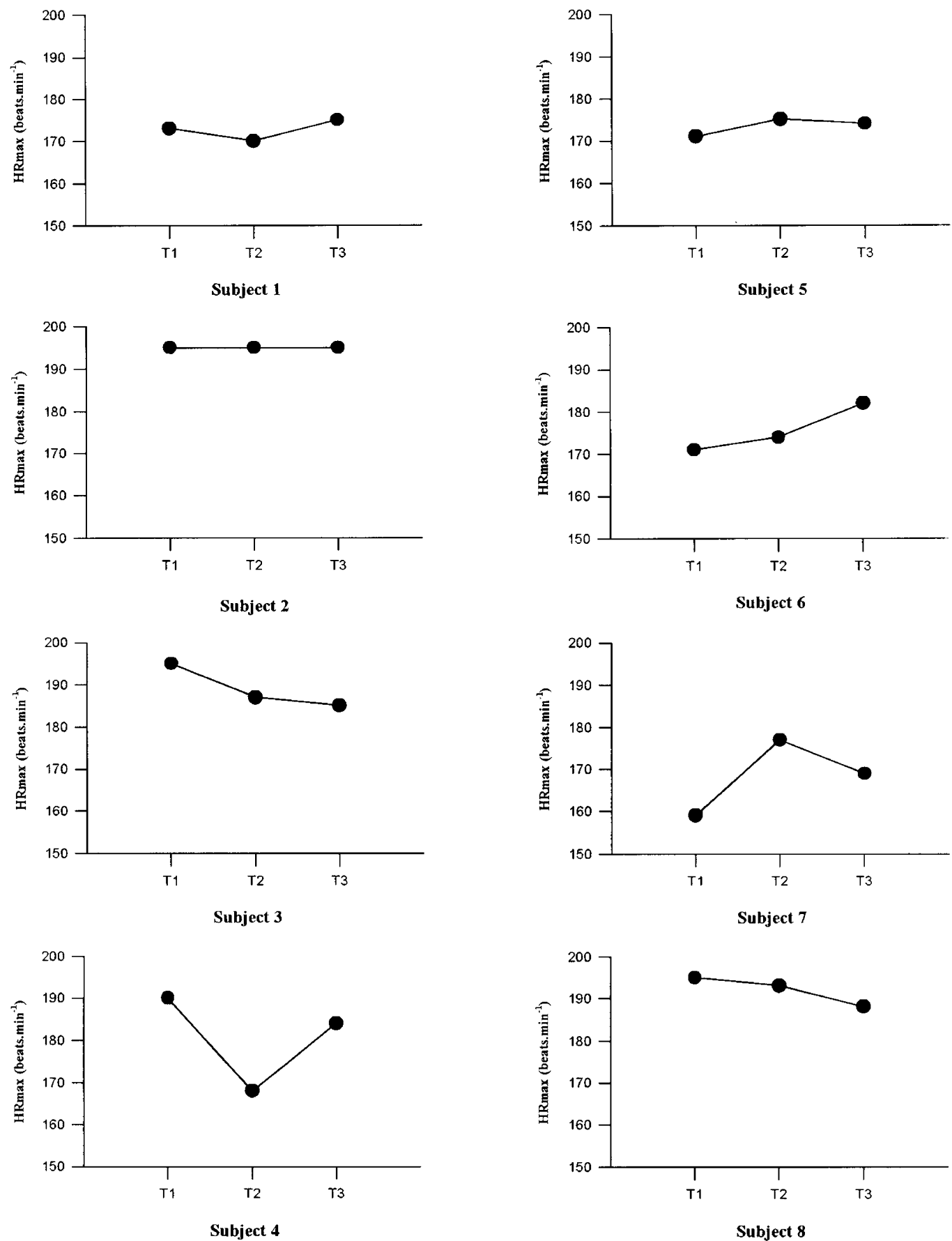

Figure 2 Maximal heart rate (HRmax in beats $\min ^{-1}$ ) measured during the three tests in each subject 
three tests for either Smax or HRmax $(F=0.08$, $P=0.92$ and $F=0.14, P=0.87$, respectively). Figures 1 and 2 present the maximal, data for all subjects.

The Bland and Altman ${ }^{13}$ procedure (Figures 3 and 4) defined the short term (STR) and the long term (LTR) reproducibility. This analysis indicated good reproducibility for Smax and HRmax between the three tests in terms of the means of difference, with no problem of proportionality between the values of the variables and their differences. The mean differences of Smax were $-0.34 \pm 0.8 \mathrm{~km} \mathrm{~h}^{-1} \quad$ (STR) and $-0.54 \pm 0.75 \mathrm{~km} \mathrm{~h}^{-1}$ (LTR) (Table 3). For HRmax, the mean differences were $1.2 \pm 11.3$ beats $\min ^{-1}$ (STR) and $-0.3 \pm 7.7$ beats $\min ^{-1}$ (LTR).
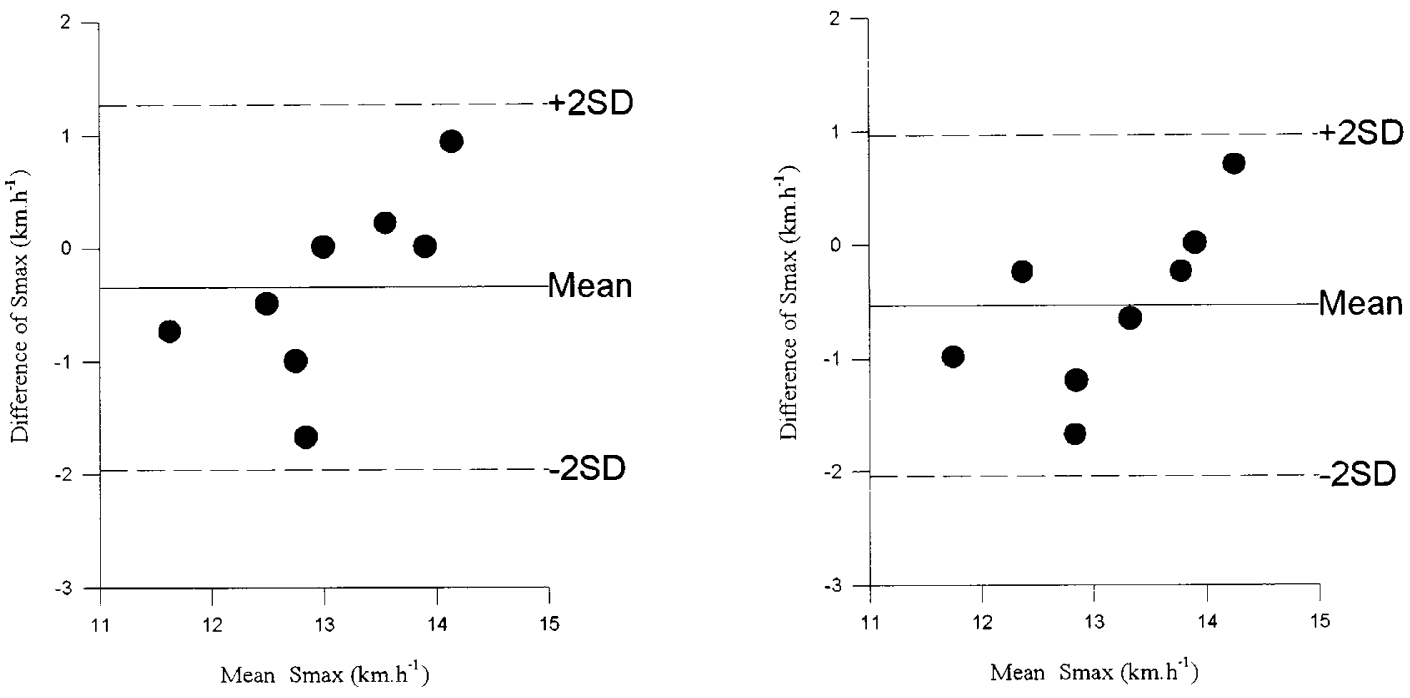

Figure 3 Comparison of maximal speed ( $\operatorname{Smax}$ in $\mathrm{km} \mathrm{h}^{-1}$ ) for first and second, and first and third tests. (a) Comparison of Smax for first and second tests (STR). (b) Comparison of Smax for first and third tests (LTR)
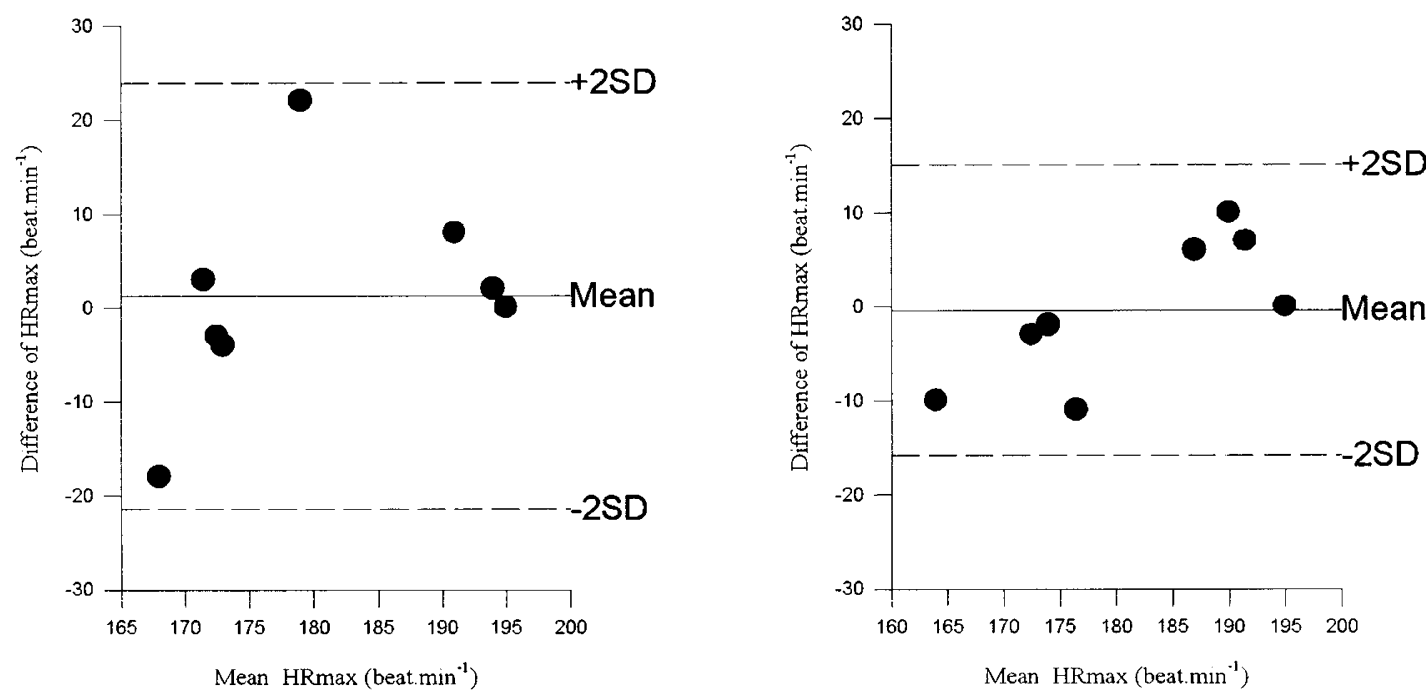

Figure 4 Comparison of maximal heart rate (HRmax in beats $\min ^{-1}$ ) for first and second, and first and third tests. (a) Comparison of HRmax for first and second tests (STR). (b) Comparison of HRmax for first and third tests (LTR) 
Table 3 Individual Smax and variability coefficients for the three tests

\begin{tabular}{lcccc}
\hline & \multicolumn{3}{c}{ Smax $\left(\mathrm{km} \cdot \mathrm{min}^{-1}\right)$} & Var coef \\
Subjects & First test & Second test & Third test & Smax \\
\hline 1 & 13 & 13 & 13 & 0.01 \\
2 & 13 & 13 & 13 & 0 \\
3 & 14 & 13 & 13 & 0.02 \\
4 & 14 & 13 & 13 & 0.05 \\
5 & 11 & 12 & 12 & 0 \\
6 & 12 & 13 & 13 & 0.02 \\
7 & 12 & 13 & 13 & 0.04 \\
8 & 12 & 12 & 12 & 0.01 \\
& 12.62 & 12.75 & 12.75 & 0.01 \\
Mean & 1.06 & 0.46 & 0.46 & 0.01 \\
SD & 1.06 & & &
\end{tabular}

Var coef $=$ variability coefficient

cord-injury athletes. All subjects reached their HRmax, which confirmed that the ALBT is a maximal test. As often reported, the individual values overall reflected the principle of inverse relationship between HRmax and lesion level inferior to T6. ${ }^{16}$ Nevertheless, some of our subjects presented the contrary. These unusual values may have been due to the different neurological and neuro-anatomical factors that affect medullary functioning, such as complete or incomplete and spastic or flaccid lesions. ${ }^{17}$ Age, athletic activity, training volume, and the number of years of athletic training can also strongly influence HRmax and, although high and complete paraplegia presents lower HRmax, this last may increase with athletic activity and training volume. ${ }^{16,18}$

Determining the reproducibility of a test is one of the steps of validation. HRmax was chosen to confirm the reproducibility of the maximal character of the ABLT. Smax was used as a potential predictive variable of $\mathrm{VO}_{2} \max$ in WD athletes, as was done in able-bodied subjects during performance of the original Leger and Boucher test. The field test of WD athletes proposed in the present study showed good reproducibility for both variables, and the simplicity of the protocol may explain this. The ALBT is an incremental and continuous field test, and the imposed pace facilitates test performance for the subjects. Although the subjects performed the test three times, which may have induced a learning effect, the Smax variability between tests was less than $1 \mathrm{~km} \mathrm{~h}^{-1}$ for all athletes. These stable low values of variability therefore indicate that there was no bias due to learning effect in the assessment of maximal variables.

Subjects did not need to employ a particular technique to propel their wheelchairs during testing. All subjects had been wheelchair-dependent for more than 2 years, and had acquired good control of them. It therefore seems likely that this population's experience and ease with their personal wheelchairs also had an influence on the reproducibility coefficients and physiological responses that we obtained. Lastly, the good reproducibility may in part be explained by the use of personal general-purpose wheelchairs rather than a standardized chair. Indeed, each wheelchair was specifically adjusted to each paraplegic, and thus was a function of very specific characteristics, such as lesion level, lesion character, length of arm, athletic activity, and so on. ${ }^{19-21}$ The differences in HRmax between tests were greater than those observed in Smax, ie, four subjects presented standard deviations of more than 5 beats min. ${ }^{-1}$ The greatest variability was observed for STR. This variability was not due to wind speed because this parameter was carefully controlled and, moreover, there was no proportionality between SD and wind speed. Stress, however, may explain this relatively high variability. We noted that some of the subjects began the first test session with resting HR values that had begun to increase. It seems quite likely that lack of familiarity with the test, and thus uncertainty about personal performance capabilities, may have been experienced as stressful. The resulting higher resting HR would have influenced the $\mathrm{HR} / \mathrm{VO}_{2}$ relationship. However, it is important to note that this greater HRmax variability between the first and second tests had no effect on Smax variability. Stress therefore did not affect Smax and differences in HRmax between tests would have no influence on the prediction of $\mathrm{VO}_{2}$ max.

\section{Conclusion}

Maximal values of heart rate and speed were reached with the adapted Leger and Boucher test for wheelchair-dependent athletes. Moreover, short- and longterm reproducibility were verified. The advantages of this field test include the simplicity of its protocol for both athlete and trainer, and its modest time and equipment requirements. The test uses only two variables, HRmax and Smax, and can be administered by trainers, athletics clubs and rehabilitation centers to adapt training programs with the goal of improving physical fitness and the athletic performances of paraplegics.

The next step will be to define a predictive equation for $\mathrm{VO}_{2} \max$ for this population. This equation will need to take into account variables such as the energy cost of wheelchair propulsion.

\section{References}

1 Vinet A et al. Validation of an incremental field test for the direct assessment of peak oxygen uptake in wheelchair-dependent athletes. Spinal Cord 1996; 34: $288-293$.

2 Léger L, Boucher R. An indirect continuous running multistage field test: the University of Montral track test. Can J of Appl Spt Sci 1980; 5: $77-84$

3 Bar-Or O and Zwiren LD. Maximal oxygen consumption test during arm exercise-reliability and validity. $J$ of Appl Physiol 1975; 38: $424-426$.

4 Glaser RM. Arm exercise training for wheelchair users. Med Sci Sports Exerc 1989; 21: $149-158$. 
5 Gass GC, Camp EM. Physiological characteristics of trained Australian paraplegic and tetraplegic subjects. Med Sci Sports Exerc 1979; 11: 256-265.

6 Lundberg A. Wheelchair driving: evaluation of a new training outfit. Scan J Rehabil Med 1980; 12: 67-72.

7 Franklin BA et al. Field test estimation of maximal oxygen consumption in wheelchair users. Arch Phys Med Rehabil 1990; 71: $574-578$.

8 Rhodes EC, McKenzie CD, Coutts KD, Rogers AR. A field test for the prediction of aerobic capacity in male paraplegics and quadraplegics. Can J Appl Spt Sci 1981; 6: 182-186.

9 MacCann B. Classifiction of the locomoter disabled for competitive sports: theory and practice. Int J Sports Med 1984; 5: $167-170$.

10 Léger L, Rouillard M. Speed reliability of cassette and tape players. Can J Appl Spt Sci 1983; 8: 47-48.

11 Sjödin B, Svendenhag J. Applied physiology of marathon running. Sports Med 1985; 2: 83-99.

12 Lange-Anderson K, Shepard RJ, Denolin H. Fundamentals of Exercise Testing. Geneva, 1971; World Health Organization.

13 Bland JM, Altman DG. Statistical methods for assessing agreement between two methods of clinical measurement. The Lancet 1986; 1: $307-310$.
14 Cooper RA et al. Maximal exercise responses of paraplegic wheelchair road racers. Paraplegia 1992; 30: 573 - 581.

15 Coutts K, Rhodes EC, McKenzie DC. Maximal exercise responses to tetraplegics and paraplegics. J Appl Physiol 1983; 55: $479-482$

16 Davis GM. Exercise capacity of individuals with paraplegia. Med Sci Sports Exerc 1993; 25: 423 - 432.

17 Van Loan MD, McCluer S, Loftin JM, Boileau RA. Comparison of physiological responses to maximal arm exercise among ablebodied, paraplegics and quadraplegics. Paraplegia 1987; 25: $397-405$.

18 Veeger HEJ et al. Peak oxygen uptake and maximal power output of Olympic wheelchair-dependent athletes. Med Sci Sports Exerc 1991; 23: $1201-1209$.

19 Bambhani N, Ertksson P, Steadward D. Reliability of peak physiological responses during wheelchair ergometry in persons with spinal cord injury. Arch Phys Med Rehabil 1991; 72: 559 562 .

20 Bernard PL. Ergonomie du déplacement en fauteuil roulant chez le traumatisé médullaire. Revue STAPS 1994; 39: 77-93.

21 Van Der Woude LHV, Veeger DJ, Rozendal RH, Sargeant TJ. Seat height in handrim wheelchair propulsion. $J$ Rehabil Research Dev 1989; 26: 31 - 50 . 